\title{
Smoluchowski approach for three-body reactions in one dimension
}

\author{
G. Oshanin, ${ }^{*}$ A. Stemmer, S. Luding, ${ }^{\dagger}$ and A. Blumen \\ Theoretical Polymer Physics, University of Freiburg, Rheinstrasse 12, 79104 Freiburg, Germany
}

(Received 5 December 1994; revised manuscript received 14 August 1995)

\begin{abstract}
We present an extension of the Smoluchowski approach for the analysis of diffusion-limited threebody reactions in one-dimensional systems. In terms of this approach we define the evolution of the particles' mean concentration over the entire time domain.

PACS number(s): 02.50. $-\mathrm{r}$, 82.20.Mj, 05.40. $+\mathrm{j}$, 05.70.Ln
\end{abstract}

\section{INTRODUCTION}

Within recent years there has been much interest [1-3] in the kinetics of $n$-body diffusion-limited reactions

$$
A+A+\cdots+A \quad(n \text { terms }) \rightarrow 0
$$

and particularly in how accurate are descriptions in terms of the conventional rate equations

$$
\frac{d C(t)}{d t}=-k(t) C^{n}(t),
$$

where $C(t)$ denotes the mean concentration of $A$ particles at time $t$ and $k(t)$ is the corresponding rate coefficient.

Most effort in this field has been invested in the understanding of bimolecular $(n=2)$ reaction kinetics [1-3]. It was realized (see, e.g., Refs. [4] and [5]) that the rate equation (2) with $n=2$ yields a qualitatively correct description of the $C(t)$ evolution, provided that the rate coefficient $k(t)$ is calculated in terms of the mean-field Smoluchowski approach (SA) [6].

The behavior of the Smoluchowski rate coefficient (SR) turns out to be very sensitive to the spatial dimensionality $d$ of the system in which the reaction takes place. In three dimensions (3D) $k(t)$ tends to a constant value, proportional to the particles' diffusion constant $D_{A}$ and to the reaction radius $R$. Consequently, at long times in 3D $C(t)$ decreases as an inverse power of $D_{A} R t$ [6]. In 1D the SR follows [3,7] $k(t) \propto\left(D_{A} / t\right)^{1 / 2}$ as $t \rightarrow \infty$ and thus $C(t) \propto 1 /\left(D_{A} t\right)^{1 / 2}$. The exact solution of the 1D reaction [7] shows that the SA predicts an adequate qualitative dependence on the pertinent parameters, $D_{A}, R$, and $t$, while the prefactors have to be somewhat modified. Finally, 2D is the marginal case, for which the SR vanishes logarithmically, $k(t) \propto D_{A} / \ln \left(D_{A} t / R^{2}\right)$, and hence $C(t)$ displays logarithmic corrections to the conventional $1 / t$ dependence, $C(t) \propto \ln \left(D_{A} t / R^{2}\right) / D_{A} t[3,8]$. Explicit forms for the SR were found also for bimolecular reactions taking place in fractal systems and in the case of particles whose motion was not diffusive (see, e.g., Refs. $[1,2])$.

\footnotetext{
*Present address: Laboratoire de Physique Théorique des Liquides, Univérsité Pierre et Marie Curie, Tour 16, 4 Place Jussieu, 75252 Paris, France.

†Present address: Laboratoire AOMC, Université Pierre et Marie Curie, Tour 13, 4 Place Jussieu, 75252 Paris, France.
}

For higher-order reaction schemes, e.g., three-body reactions $(n=3)$, the case is less transparent. Most work pertains to complex chemical situations involving threebody reactions as an elementary step and focuses on the analysis of bifurcation structures, such as multistable states, oscillations, chemical waves, and chemical chaos [9-12]. The kinetics of diffusion-limited three-body reactions has been less studied. The question of the effects of particle diffusion on the kinetics of three-body reactions was first addressed in Ref. [13]. This work, and also the recent Ref. [14], aimed at finding an analog to the SA for 3D three-body reactions. In Ref. [15] several general statements on the time evolution of $n$-body reactions were made using scaling arguments. Thus for $n$-body reactions taking place in $d$-dimensional systems there should exist [15] an upper critical dimension $d_{c}(n)=2 /(n-1)$. Above $d_{c}(n), d>d_{c}(n), C(t)$ follows

$$
C(t) \propto\left[\frac{1}{k t}\right)^{1 /(n-1)},
$$

with $k$ being time independent (but as yet an unspecified function of the pertinent parameters). For $d<d_{c}(n)$ [which is more of conceptual than of physical interest, since $d_{c}(n)$ does not exceed unity already for three-body reactions involving diffusive particles], a power-law decay, $C(t) \propto t^{-d / 2}$, has been predicted. Finally, for the marginal case $d=d_{c}(n)$ one expects logarithmic corrections to Eq. (3) [15]. Thus for three-body reactions in 1D one expects $k(t)$ in Eq. (2) to vanish as $1 / \ln (t)$ [16], so that

$$
C(t) \propto\left[\frac{\ln (t)}{t}\right]^{1 / 2} .
$$

This behavior was meanwhile observed in numerical simulations [17] of reactions $3 A \rightarrow A$ and $3 A \rightarrow 2 A$ and was explained through simple physical arguments [18], similar to the ideas of Ref. [13], and also through a renormalization-group analysis [19].

In the present paper we start developing the SA for diffusion-limited $n$-body reactions, focusing on threebody reactions in $1 \mathrm{D}$. We determine here the dependence of $k(t)$ and thus of $C(t)$ on the pertinent parameters for the whole time regime; we note that previous work considered mainly the long-time asymptotics of $C(t)$. As we proceed to show, Eq. (4) arises naturally in terms of an appropriate extension of the SA; the origin of the loga- 
rithmic correction in Eq. (4) is essentially the same as that for the bimolecular reactions in 2D. The formalism allows us to determine $C(t)$ for 1D three-body reactions when the reacting particles move subdiffusively. Our analytical predictions are confirmed by the results of numerical simulations.

\section{THE MODEL AND BASIC EQUATIONS}

Consider a one-dimensional regular lattice of spacing $a$, which is partially, with mean concentration $C_{0}$, occupied by particles $A$. Each $A$ particle performs a symmetric random walk: being at site $x$ it jumps with probability $\frac{1}{2}$ to one of the adjacent sites, $x-a$ or $x+a$. The time interval between the successive jumps has an exponential distribution with parameter $\tau$. The hard-core exclusion between $A$ particles is disregarded and thus the random walks of the particles are independent of each other.

The particles undergo chemical reactions: whenever the positions $x_{1}, x_{2}$, and $x_{3}$, of any three $A$ particles satisfy

$\left|x_{1}-x_{2}\right| \leq R, \quad\left|x_{1}-x_{3}\right| \leq R$, and $\left|x_{2}-x_{3}\right| \leq R$,

where $R$ is the reaction radius, this triple reacts instantly and all three particles are removed from the system.

Let $C(x, t)$ be the local concentration of $A$ particles at site $x$ at time $t$. Its evolution due to the reaction and to the random walk is described in the continuous time limit by

$$
\begin{aligned}
\dot{C}(x, t)= & -\frac{k_{c}}{3} \sum_{\Omega(R)} C(x, t) C\left(x+\lambda_{1}, t\right) C\left(x+\lambda_{2}, t\right) \\
& +\frac{1}{2 \tau} \Delta(x) C(x, t)
\end{aligned}
$$

where $\Delta(x)$ is the central difference operator, $\Delta(x) \equiv \nabla_{+}(x)-\nabla_{-}(x)$, with

$$
\nabla_{ \pm}(x) C(x, t)= \pm[C(x \pm a, t)-C(x, t)],
$$

so that

$$
\Delta(x) C(x, t)=[C(x-a, t)+C(x+a, t)-2 C(x, t)],
$$

and $\Omega(R)$ denotes the reaction zone of site $x$. This includes all the points in the $\left(\lambda_{1}, \lambda_{2}\right)$ plane, whose coordinates $\left(\lambda_{1}, \lambda_{2}\right)$ satisfy the inequalities in Eqs. (5); $\Omega(R)$ is a hexagon (see Fig. 1), centered on the line $\lambda_{1}=\lambda_{2}$.

The property of interest is the mean concentration,

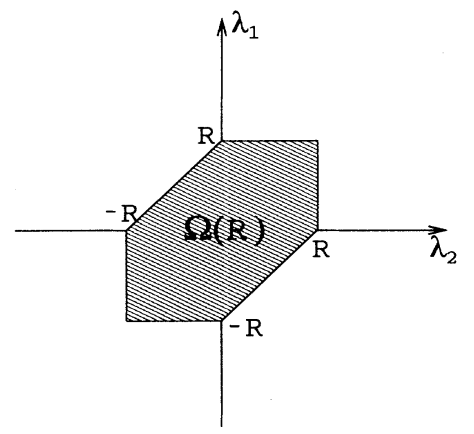

FIG. 1. The reaction zone $\Omega(R)$, defined by the inequalities in Eqs. (5).

$$
C(t)=\frac{1}{V} \sum_{x} C(x, t)
$$

where $V$ stands for the volume of the system and $C(t=0)=C_{0}$. The mean concentration obeys

$$
\dot{C}(t)=-\frac{k_{c}}{3} \sum_{\Omega(R)} T\left(\lambda_{1}, \lambda_{2}\right),
$$

where $T\left(\lambda_{1}, \lambda_{2}\right)$ denotes the three-particle correlation function

$$
T\left(\lambda_{1}, \lambda_{2}\right)=\frac{1}{V} \sum_{x} C(x, t) C\left(x+\lambda_{1}, t\right) C\left(x+\lambda_{2}, t\right) .
$$

The rate coefficient $k_{c}$ in Eqs. (6) and (9) describes the probability of an annihilation of a triple of $A$ particles, whose positions are within the same reaction zone; in our consideration the annihilation of such a triple takes place with unit probability and in the subsequent calculations we will set $k_{c} \rightarrow \infty$. Finally, the factor $\frac{1}{3}$ in Eqs. (6) and (9) is introduced due to the following reasoning. Consider a triple of $A$ particles, whose positions are $x, x-R / 2$, and $x+R / 2$. One may notice that this triple belongs to the reaction zones of the sites $x, x-R / 2$, and $x+R / 2$, and thus enters the right-hand side (RHS) of the equations for the evolution of the local concentrations at these sites. Consequently, in $C(t)$, Eq. (8), the reaction event in this triple will be counted three times as an independent contribution of three different sites. Therefore the factor $\frac{1}{3}$ prevents an overestimating of the total number of reaction events.

Consider now the evolution of the third-order correlations. Taking the time derivative of $T\left(\lambda_{1}, \lambda_{2}\right)$ in Eq. (10) and making use of Eq. (6) we obtain

$$
\begin{aligned}
\dot{T}\left(\lambda_{1}, \lambda_{2}\right)=\frac{1}{2 \tau}[ & T\left(\lambda_{1}+a, \lambda_{2}+a\right)+T\left(\lambda_{1}-a, \lambda_{2}-a\right)+T\left(\lambda_{1}+a, \lambda_{2}\right)+T\left(\lambda_{1}-a, \lambda_{2}\right)+T\left(\lambda_{1}, \lambda_{2}+a\right) \\
& \left.+T\left(\lambda_{1}, \lambda_{2}-a\right)-6 T\left(\lambda_{1}, \lambda_{2}\right)\right]-F\left(\lambda_{1}, \lambda_{2}\right),
\end{aligned}
$$

where $F\left(\lambda_{1}, \lambda_{2}\right)$ stems from the nonlinear reaction terms in Eq. (6) and is given explicitly by 


$$
\begin{gathered}
F\left(\lambda_{1}, \lambda_{2}\right)=\frac{k_{c}}{3 V} \sum_{\Omega(R)} \sum_{x} C(x, t) C\left(x+\lambda_{1}, t\right) C\left(x+\lambda_{2}, t\right) \\
\times\left[C\left(x+\lambda_{1}, t\right) C\left(x+\lambda_{2}, t\right)+C\left(x+2 \lambda_{1}, t\right) C\left(x+\lambda_{1}+\lambda_{2}, t\right)\right. \\
\left.+C\left(x+2 \lambda_{2}, t\right) C\left(x+\lambda_{1}+\lambda_{2}, t\right)\right]
\end{gathered}
$$

i.e., $F\left(\lambda_{1}, \lambda_{2}\right)$ is proportional to the fifth power of the particles' concentration. Making use of the forward and backward difference operators in Eq. (7) we can recast Eq. (11) into the more compact form. Noticing that

$$
\begin{gathered}
T\left(\lambda_{1}+a, \lambda_{2}+a\right)+T\left(\lambda_{1}-a, \lambda_{2}-a\right)-2 T\left(\lambda_{1}, \lambda_{2}\right) \\
=\left[\nabla_{-}\left(\lambda_{1}\right) \nabla_{-}\left(\lambda_{2}\right)+\nabla_{+}\left(\lambda_{1}\right) \nabla_{+}\left(\lambda_{2}\right)\right. \\
\left.\quad+\Delta\left(\lambda_{1}\right)+\Delta\left(\lambda_{2}\right)\right] T\left(\lambda_{1}, \lambda_{2}\right)
\end{gathered}
$$

we have

$$
\begin{aligned}
\dot{T}\left(\lambda_{1}, \lambda_{2}\right)=\frac{1}{\tau}[ & \frac{1}{2} \nabla_{-}\left(\lambda_{1}\right) \nabla_{-}\left(\lambda_{2}\right)+\frac{1}{2} \nabla_{+}\left(\lambda_{1}\right) \nabla_{+}\left(\lambda_{2}\right) \\
& \left.+\Delta\left(\lambda_{1}\right)+\Delta\left(\lambda_{2}\right)\right] T\left(\lambda_{1}, \lambda_{2}\right)-F\left(\lambda_{1}, \lambda_{2}\right) .
\end{aligned}
$$

\section{MODIFIED SMOLUCHOWSKI APPROACH FOR THREE-BODY REACTIONS IN $1 D$}

We proceed further by recalling the Collins and Kimball analysis [20] of bimolecular reaction kinetics. It was shown in Ref. [20] that the Smoluchowski form of the rate coefficient can be obtained from the hierarchy of equations obeyed by the correlation functions. The derivation involves two basic assumptions. The first one is the truncation of the hierarchy; in the case of bimolecular reactions, when the reaction rate depends on the pair correlations, the hierarchy was truncated at the level of third-order correlations. Second, it was assumed that the pair correlations obey the radiation boundary condition in the reaction zone. Making use of the Gauss divergence theorem, one finds [20] then that the reaction rate equals the flux of particles into the reaction zone. The Smoluchowski form of the reaction rate appears naturally in such considerations when the limit $k_{c} \rightarrow \infty$ is taken.

Following the lines of Ref. [20] we assume that in the case of three-body reactions the hierarchy of correlation functions can be truncated at the level of fifth-order correlations, i.e., we set $F\left(\lambda_{1}, \lambda_{2}\right)=0$. Then, Eq. (14) [or Eq. (11)] becomes closed with respect to $T\left(\lambda_{1}, \lambda_{2}\right)$. It might be useful to note that it describes a random walk of a particle on a triangular lattice, the distribution of the time intervals between successive jumps being exponential, with a mean waiting time of $\tau / 3$. Further on, taking the limit $k_{c} \rightarrow \infty$ we have

$$
\left.T\left(\lambda_{1}, \lambda_{2}\right)\right|_{\lambda_{1}, \lambda_{2} \in \Omega(R)}=0,
$$

i.e., Smoluchowski's absorbing boundary condition. The reaction rate in Eq. (9) is then given by

$$
k_{c} \sum_{\Omega(R)} T\left(\lambda_{1}, \lambda_{2}\right)=\sum_{\Gamma(R+a)} h T\left(\lambda_{1}, \lambda_{2}, t\right),
$$

where the sum on the RHS extends over all sites of the perimeter $\Gamma(R+a)$ of the hexagon defined by Eqs. (5) with $R$ replaced by $R+a$. The weight factor $h$ occurs due to the topology of the triangular lattice; it equals $\frac{1}{2}$ for the vertex sites and 1 for any other site of the perimeter $\Gamma(R+a)$.

Furthermore, Eq. (14) is complemented by two traditional boundary conditions $[6,20]$. The first is that at $t=0$ the particles are uniformly distributed on the lattice, i.e.,

$$
\left.T\left(\lambda_{1}, \lambda_{2}\right)\right|_{t=0}=C_{0}^{3} .
$$

The second is that at large separations from the reaction zone the correlations decouple, i.e.,

$$
\left.T\left(\lambda_{1}, \lambda_{2}\right)\right|_{\lambda_{1}, \lambda_{2} \pm \infty}=C^{3}(t) \text {. }
$$

Now the system of reaction-diffusion equations $(14)-(18)$ is closed and to solve it we turn to the continuous-space limit. Equation (14) then takes the form

$$
\dot{T}\left(\lambda_{1}, \lambda_{2}\right)=2 D_{A}\left[\frac{\partial^{2}}{\partial \lambda_{1}^{2}}+\frac{\partial^{2}}{\partial \lambda_{2}^{2}}+\frac{\partial^{2}}{\partial \lambda_{1} \partial \lambda_{2}}\right] T\left(\lambda_{1}, \lambda_{2}\right)
$$

where $D_{A}$ is the diffusion constant, $D_{A}=a^{2} / 2 \tau$. Equation (19) is to be solved subject to the boundary and initial conditions in Eqs. (15)-(18). Let us note that in the discrete-space description the random walk, Eq. (14), and the boundary condition in Eq. (15) happen to have the same symmetry. In the continuum we have the elliptic differential Eq. (19) and to preserve the symmetry between the differential operator and the shape of the boundary we approximate the hexagonal trap by the ellipse

$$
\frac{3}{4}\left(\lambda_{1}-\lambda_{2}\right)^{2}+\frac{1}{4}\left(\lambda_{1}+\lambda_{2}\right)^{2}=R^{2} .
$$

We note that the replacement of the piecewise boundary by the ellipse may, of course, incur some errors in the reaction rate. We believe, however, that this approximation affects only the short-time kinetics and has no influence on the long-time behavior. This is also supported by the recent results of Ref. [21], which show that the precise form of the trapping boundary plays no role at large times in $2 \mathrm{D}$, the only relevant parameter being the 
maximal linear extension of the trap. Approximating the hexagon Eqs. (5) by the ellipse Eq. (20) preserves this property.

It is expedient now to use polar coordinates $(\rho, \phi)$, such that $\rho=\left(\lambda_{1}^{2}+\lambda_{2}^{2}-\lambda_{1} \lambda_{2}\right)^{1 / 2}$ and $\phi=\operatorname{arctanh}\left[\sqrt{3}\left(\lambda_{1}\right.\right.$ $\left.\left.-\lambda_{2}\right) /\left(\lambda_{1}+\lambda_{2}\right)\right]$. In these coordinates the ellipse Eq. (20) transforms into a circle of radius $R$ and Eq. (19) takes the form of a $2 \mathrm{D}$ diffusion equation

$$
\dot{T}(\rho)=\frac{3 D_{A}}{2}\left[\frac{\partial^{2}}{\partial \rho^{2}}+\frac{1}{\rho} \frac{\partial}{\partial \rho}\right] T(\rho) .
$$

The angular part of the diffusion operator is omitted since now the boundaries, and thus $T(\rho)$, are independent of the variable $\phi$.

Therefore the definition of the reaction rate in our case, apart from the renormalization of the diffusion constant, becomes identical to the definition of the SR for bimolecular reactions in 2D. The formal solution thus follows (see Ref. [22])

$$
\begin{aligned}
T(\rho)=\frac{2 C^{3}(t)}{\pi} & \int_{0}^{\infty} \frac{d z}{z} \exp \left[-\frac{3 D_{A} t z^{2}}{2 R^{2}}\right) \\
& \times \frac{J_{0}(z) Y_{0}(\rho z / R)-J_{0}(\rho z / R) Y_{0}(z)}{J_{0}^{2}(z)+Y_{0}^{2}(z)},
\end{aligned}
$$

where $J_{0}(z)$ and $Y_{0}(z)$ denote the Bessel functions of the first and the second kind, respectively.

Now from Eqs. (9) and (16) we find that the mean concentration $C(t)$ obeys Eq. (2) with $n=3$ and with $k(t)$ defined by

$$
k(t)=\frac{8 D_{A}}{\pi \sqrt{3}} \int_{0}^{\infty} \frac{d z}{z} \frac{\exp \left(-3 D_{A} t z^{2} / 2 R^{2}\right)}{J_{0}^{2}(z)+Y_{0}^{2}(z)} .
$$

Equation (23) determines the evolution of $k(t)$ over the entire time domain. From the analysis of Ref. [8] it follows that $k(t)$ in Eq. (23) is a slowly varying function and it suffices to consider its asymptotic behavior only. The large- $t$ expansion of the integral in Eq. (23) was presented in Refs. [8] and [22] and yields for the rate coefficient in our case

$$
k(t)=\frac{4 \pi D_{A}}{\sqrt{3} y(t)}\left[1-\frac{\gamma}{y(t)}-\frac{\left(\pi^{2} / 6-\gamma^{2}\right)}{y^{2}(t)}-\cdots\right] .
$$

In Eq. (24), $\gamma=0.577 \ldots$ is Euler's constant and $y(t)=\ln \left(6 D_{A} t / R^{2}\right)-2 \gamma$. From Eq. (2) we have

$$
C(t)=C_{0}\left\{1+2 C_{0}^{2} \int_{0}^{t} d t^{\prime} k\left(t^{\prime}\right)\right\}^{-1 / 2},
$$

where for $t$ large

$$
\begin{aligned}
\int_{0}^{t} d t^{\prime} k\left(t^{\prime}\right)= & \frac{4 \pi D_{A} t}{\sqrt{3} y(t)} \\
& \times\left[\begin{array}{c}
1+\frac{1-\gamma}{y(t)}-\frac{2(\gamma-1)-\gamma^{2}+\pi^{2} / 6}{y^{2}(t)} \\
\\
+\cdots] .
\end{array}\right.
\end{aligned}
$$

For $t \rightarrow \infty$ the logarithmic corrections in the brackets in Eq. (26) can be neglected. Thus at long times the leading behavior of $C(t)$ is

$$
C(t) \propto\left[\frac{\ln \left(\beta D_{A} t / R^{2}\right)-2 \gamma}{\alpha D_{A} t}\right]^{1 / 2},
$$

with $\alpha=8 \pi / \sqrt{3}$ and $\beta=6$. Equation (27) obeys the asymptotic form of Eq. (4). We note, however, that the numerical factors in Eq. (27) differ from the earlier reported values $\alpha=8 \pi, \beta=2$ [18] and $\alpha=4 \pi \sqrt{3}, \beta=1[19$ ].

Motivated by this discrepancy, we have performed numerical simulations of three-body reactions on a 1D lat-

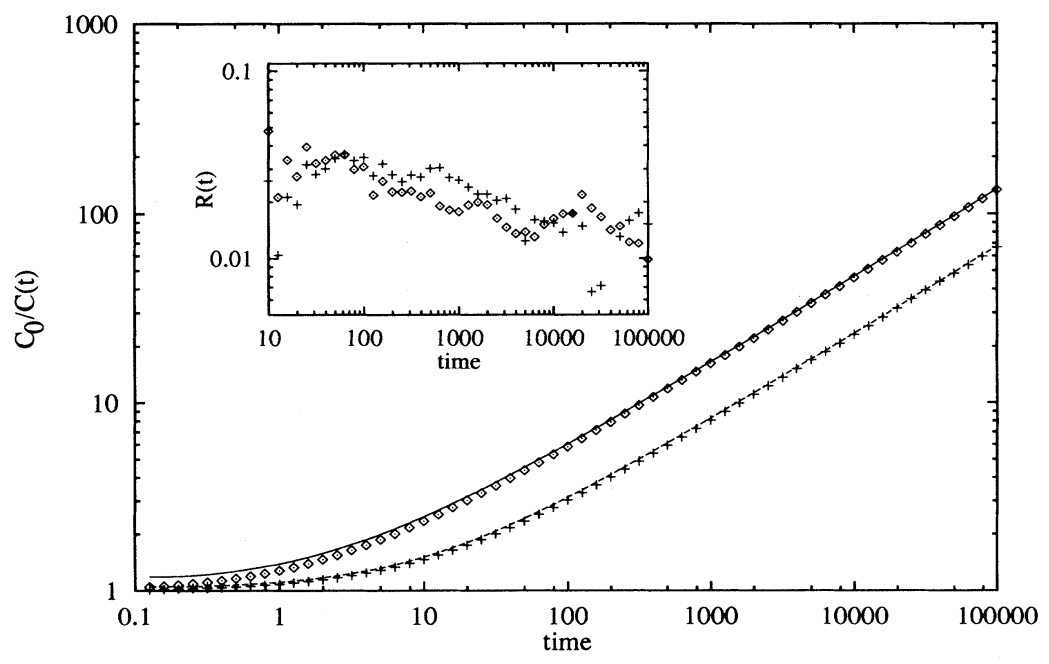

FIG. 2. Log-log plot of $C_{0} / C(t)$ as a function of time. The diamonds represent the numerical data for $C_{0}=0.6$ and the crosses the data for $C_{0}=0.3$. The lines give our analytical results, Eqs. (25) and (26), for $C_{0}=0.6$ (solid line) and $C_{0}=0.3$ (dashed line). The inset displays the deviation $R(t)=\left[C_{\text {an }}(t)\right.$ $\left.-C_{\text {num }}(t)\right] / C_{\text {an }}(t)$, where $C_{\text {an }}(t)$ denotes the analytical result of Eqs. (25) and (26) and $C_{\text {num }}(t)$ the simulation results. 

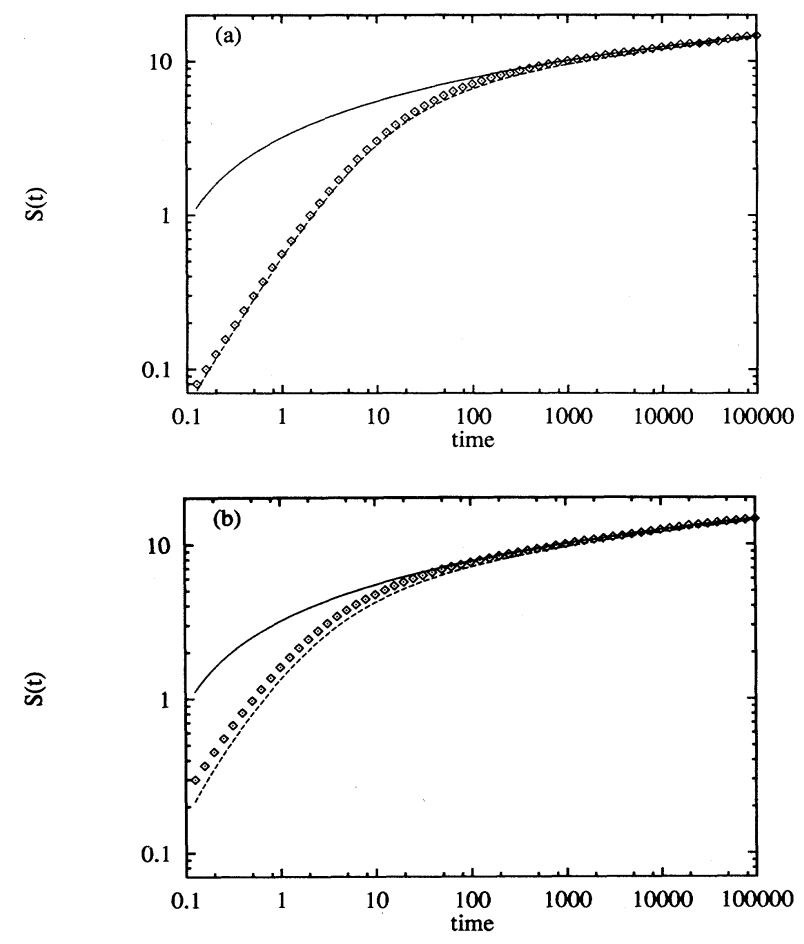

FIG. 3. Evidence for logarithmic corrections. Here we display a $\log$-log plot of $S(t)=\alpha D_{A} t C^{2}(t)$ as a function of time. The diamonds are the simulation results for (a) $C_{0}=0.3$ and (b) $C_{0}=0.6$. Here $S(t)$ is plotted as a dashed line with $C(t)$ given by Eqs. (25) and (26) and as a solid line with $C(t)$ given by Eq. (27).

tice with $5 \times 10^{5}$ sites and periodic boundary conditions. For a given mean concentration $C_{0}$ the particles were initially placed at random positions on the lattice, subject to the constraint that each site supports at most double occupancy. The simulation algorithm followed the analytical model closely. In each simulation step we chose one particle at random and let this particle jump with probability $\frac{1}{2}$ either to the right or to the left, regardless of the occupancy of the adjacent site. We let $\tau$ be the mean time between successive jumps of the particle and $a$ the step length. After each jump we increase the time by $\Delta t=1 / N$, where $N$ is the number of particles present in the system; thus during $t=1$ every particle, on average, performs one step, and, taking $a=\tau=1$, the diffusion coefficient $D_{A}=a^{2} / 2 \tau$ is $\frac{1}{2}$. The reaction occurs immediately when a particle jumps onto a doubly occupied site; then all three particles involved are removed from the system. In Figs. 2 and 3 we present the results of simulations for two different initial concentrations, $C_{0}=0.6$ and $C_{0}=0.3$, respectively. In our plot we set $D_{A}=\frac{1}{2}$ and have taken $R=\exp (-\gamma) / \sqrt{8}$. This choice of $R$, as shown in Refs. [23] and [24], is the effective radius of a single adsorbing site of a $2 \mathrm{D}$ lattice in the continuum. It is instructive to note that for such a value of $R$ Eq. (26) coincides with the result of Ref. [23] for the number of distinct sites visited by a random walker on a triangular lattice. The inset in Fig. 2 shows the deviation between our analytical and numerical results, which never exceeds $2 \%$. This comparison of the numerical data with the analytical results demonstrates thus that the Smoluchowski approach, as extended here, provides a fair description of three-body diffusion-limited reactions.

\section{ACKNOWLEDGMENTS}

The authors thank Professor S. F. Burlatsky, Professor M. Moreau, Professor G. Nicolis, and Professor R. Kapral for helpful discussions. G.O. acknowledges financial support from the Alexander von Humboldt Foundation and CNRS. S.L. is supported by the European Community Project "Human Capital and Mobility." A.B. acknowledges support by the Deutsche Forschungsgemeinschaft (SFB 60), by the PROCOPE program of the DAAD, and by the Fonds der Chemischen Industrie.
[1] A. Blumen, J. Klafter, and G. Zumofen, in Optical Spectroscopy of Glasses, edited by I. Zschokke (Reidel, Dordrecht, 1986), p. 199.

[2] G. Oshanin, M. Moreau, and S. F. Burlatsky, Adv. Colloid Interface Sci. 49, 1 (1994).

[3] A. A. Ovchinnikov, S. F. Timashev, and A. A. Belyy, Kinetics of Diffusion Controlled Chemical Processes (Nova Science, New York, 1989).

[4] A. Blumen, J. Klafter, and G. Zumofen, Phys. Rev. B 27, 3429 (1983).

[5] S. F. Burlatsky and A. A. Ovchinnikov, Zh. Eksp. Teor. Fiz. 92, 1618 (1987) [Sov. Phys. JETP 65, 908 (1987)].

[6] M. von Smoluchowski, Z. Phys. B 16, 321 (1915).

[7] D. C. Torney and H. M. McConnell, Proc. R. Soc. London Ser. A 387, 147 (1983).

[8] D. C. Torney and H. M. McConnell, J. Phys. Chem. 87,
1941 (1983).

[9] G. Nicolis and I. Prigogine, Self Organization in Nonlinear Systems (Wiley, New York, 1977).

[10] C. Vidal and H. Lemarchand, La Réaction Créatrice (Hermann, Paris, 1988).

[11] R. Kapral, J. Math. Chem. 6, 113 (1991).

[12] A. Provata, J. W. Turner, and G. Nicolis, J. Stat. Phys. 70, 1195 (1992); A. Tretyakov, A. Provata, and G. Nicolis, J. Phys. Chem. (to be published).

[13] S. F. Burlatsky and A. A. Ovchinnikov, Russ. J. Phys. Chem. 54, 1741 (1980); the English translation of this paper can be found also in [3], Sec. 1.7.

[14] S. F. Burlatsky and M. Moreau, Phys. Rev. E 51, 2363 (1995).

[15] K. Kang and S. Redner, Phys. Rev. A 32, 435 (1985).

[16] V. Privman and M. D. Grynberg, J. Phys. A 25, 6567 
(1992).

[17] D. ben-Avraham, Phys. Rev. Lett. 71, 3733 (1993).

[18] P. L. Krapivsky, Phys. Rev. E 49, 3233 (1994).

[19] B. P. Lee, J. Phys. A 27, 2633 (1994).

[20] F. Collins and G. Kimball, J. Colloid Sci. 4, 425 (1949); see also T. R. Waite, Phys. Rev. 107, 463 (1957).

[21] S. F. Burlatsky, O. F. Ivanov, and J. M. Deutch, J. Chem.
Phys. 97, 156 (1992).

[22] H. S. Carslaw and J. C. Jaeger, Conduction of Heat in Solids, 2nd ed. (Oxford University Press, Oxford, 1953), p. 335.

[23] G. Zumofen and A. Blumen, J. Chem. Phys. 76, 3713 (1982).

[24] R. M. Ziff, J. Stat. Phys. 65, 1217 (1991). 